\title{
Definición y estado del arte de la ingeniería concurrente la manufactura por computer y la mecatrónica
}

\section{Definition and state of the art of concurrent engineering manufacturing by computer and mechatronics}

Estela María Macas

Universidad Internacional del Ecuador, Ecuador

Autor para correspondencia: esstela@gmail.com

Fecha de recepción: 05 de Junio de 2017 - Fecha de aceptación: 30 de Septiembre de 2017

\begin{abstract}
Resumen
El objetivo concreto de este artículo es proporcionar un enfoque explicativo de las teorías y estado del arte de Ingeniería concurrente, Manufactura y Mecatrónica; para aquellas personas con nivel de formación universitaria que, en algún momento u otro de su actividad profesional, deberán emprender el diseño o la fabricación de un producto o una máquina. En los últimos años se ha ido desarrollado una nueva forma de entender la ingeniería en la que el diseño acumula cada vez mayores responsabilidades. En efecto, estas nuevas visiones propugnan que una correcta definición y concepción global de los sistemas o máquinas debe tener en cuenta tanto los requerimientos de su ciclo de vida como la gama de productos fabricada por la empresa o sector, lo que constituye la mejor garantía para su correcto funcionamiento y su acierto comercial. Finalmente agradezco y deseo que este contenido sea del interés para los lectores.
\end{abstract}

Palabras Claves: ingeniería concurrente; manufactura; mecatrónica

\begin{abstract}
The specific objective of this article is to provide an explanatory approach to the theories and state of the art of concurrent Engineering, Manufacturing and Mechatronics; for those with a university education level who, at some point or another of their professional activity, must undertake the design or manufacture of a product or a machine. In recent years a new way of understanding engineering has been developed in which design accumulates increasing responsibilities. Indeed, these new views advocate that a correct definition and overall design of systems or machines must take into account both the requirements of their life cycle and the range of products manufactured by the company or sector, which is the best guarantee for its correct operation and its commercial success. Finally, I thank and wish that this content is of interest to readers.
\end{abstract}

Key words: concurrent engineering; manufacturing; mechatronics 


\section{Introducción}

En estos últimos años la humanidad, se ha maravillado con los inmensos progresos que la ciencia y la tecnología ha puesto al servicio del mundo, tanto en el hardware como en el software informático, hoy en día surgen nuevas tendencias conocidas como la Mecatrónica, Ingeniería Concurrente y Manufactura asistida por computador. Si bien es cierto tiene sus antecedentes; sin embargo; desde las primeras conceptualizaciones han surgido diversas definiciones que intentan describir esta disciplina en forma clara y precisa. La intencionalidad es impulsar por medio de la creatividad de innovación, escenarios y procesos que inserte a estos elementos teóricos para la optimización de productos y sistemas técnicos de complejidad reciente.

La globalización de los mercados implica una competencia cada vez más feroz. Sólo las empresas capaces de ofrecer los productos de mejor calidad en precio adecuado y en un tiempo más corto sobreviven. El reducir el tiempo de respuesta, la adecuación del producto a las necesidades del cliente, un mantenimiento eficaz, a bajo precio, un estándar de calidad y costo adecuado son los objetivos que pretende cubrir la Ingeniería Concurrente.

La Mecatrónica permite simplificar las soluciones de vida, la idea es reutilizar y crear mejoramientos en los procesos cotidianos utilizando la creatividad a través de conocimientos académicos enfocados a la mecánica y automatización de procesos para utilizar adecuadamente la tecnología combinando con la asociación de diferentes Ramas Ingenieriles.

\section{Definiciones Generales}

\section{La ingeniería concurrente}

También llamada por muchos autores como ingeniería simultánea, es un fenómeno que aparece a principios de la década de los ochenta en el Japón y que llega a Europa a través de América, fundamentalmente Estados Unidos, a finales de esa misma década.

El objetivo de una empresa industrial es, en pocas palabras: "Diseñar productos funcionales y estéticamente agradables en un plazo de lanzamiento lo más corto posible, con el mínimo coste, con el objetivo de mejorar la calidad de vida del usuario final".

Evidentemente, este objetivo se debe alcanzar dentro de la filosofía del libre mercado, donde la industria debe vivir de sus propios recursos. La ingeniería concurrente que ahora se aborda es una filosofía basada en sistemas informáticos y, como la gran mayoría de estos sistemas, su aportación fundamental consiste en una muy evolucionada forma de tratar la información disponible. Fig. 1

El término concurrente se refiere a la formación de equipos de trabajo formados por personas de diversas áreas como mercadotecnia, diseño, manufactura, finanzas, etc., con la finalidad de desarrollar y diseñar productos de acuerdo a los requerimientos de mercadotecnia, pero tomando en cuenta al personal, el equipo de manufactura, el empaque, el diseño, el tipo de materiales, etc. 


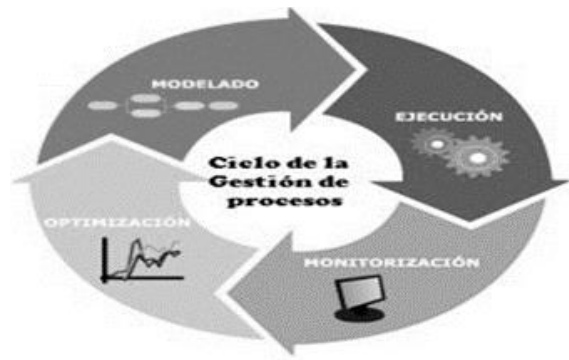

Fig. 1 Representación por procesos Fuente (LEXNOVA. 2011)

La ingeniería concurrente es la ciencia que permite la integración sistemática y simultánea del diseño de productos y procesos considerando la fabricación y su usabilidad correspondiente (servicio que brinde), siempre orientado a que el servicio sea eficiente, eficaz y óptimo. Fig. 2.

Así mismo permite que los programadores, iniciado el proyecto, tomen en cuenta los elementos preliminares que debe contener su ciclo de vida del producto apoyados de las TI con una organización flexible, esto permitirá acortar los tiempos de desarrollo del producto y elevar de gran forma su productividad.

Persigue un estudio sistemático, simultáneo, en el momento del desarrollo del producto, de las necesidades de mercado que va a cubrir, de los requisitos de calidad y costos, de los medios y métodos de fabricación, venta y servicio necesarios para garantizar la satisfacción del cliente

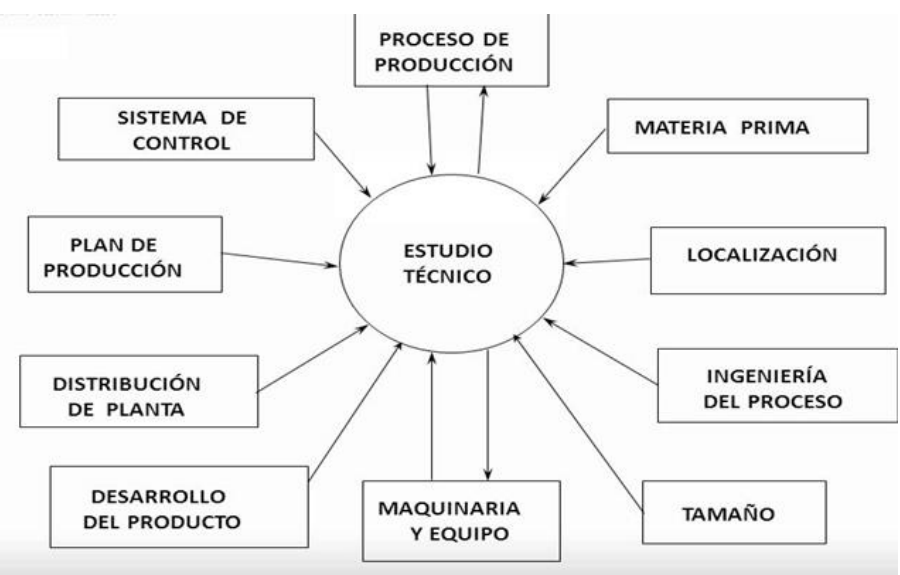

Fig. 2 Ejemplo de Ingeniería Concurrente Fuente (Juan Paucar. 2014)

La Ingeniería concurrente requiere del trabajo colaborativo de todos los miembros del equipo para generar un producto con las características deseadas en el tiempo programado.

\section{La Mecatrónica}

Acuñada en 1969 por el ingeniero japonés Yakasawa, la palabra Mecatrónica ha sido definida de varias maneras. 
Se puede hacer referencia a la definición de Mecatrónica que cita: "Mecatrónica es la combinación sinérgica de la ingeniería mecánica de precisión, de la electrónica, del control automático y de los sistemas para el diseño de productos y procesos"(J.A. Rietdijk, 1989). Fig. 3.

La Mecatrónica está dirigida a las aplicaciones y al diseño continuo. Fig. 4 permite orientar simbólicamente la Mecatrónica.

Las personas en general han permitido acelerar esta ciencia por las constantes ideas innovadoras en donde permiten conjugar la sinergia de uso de la Mecatrónica con mecánica, electrónica, computación, sistemas de control entre otros elementos, teniendo como punto de partida el análisis de la robotización, el estar conectado y crear múltiples procesos acertados para su usabilidad.

De igual forma el sistema competitivo actual apoyado de la Mecatrónica ha permitido unificar el sistema, producto y servicio para mejorar productos de servicios finales con carácter de innovación social, básicamente lo que ocurre actualmente en el mundo es una norma que hay un momento en diferenciar lo que es y lo que se pueda hacer; y hacerlo acertadamente usando el cambio tecnológico.

Todos somos conscientes que nuestro país está actualmente inmerso en un proceso de industrialización, la Mecatrónica contribuye satisfactoriamente en este entorno industrializado con la aportación de elementos que favorezcan a la reducción de costos, logro de efíciencia, mayor productividad a través de su automatización de procesos.

Dentro del desarrollo de tecnologías tenemos esta ciencia tan interesante como lo es la Mecatrónica que permite dimensionar los modelos utilizando funcionalidad, optimización creando a partir de piezas funcionales a soluciones automatizadas para la simplificación de tareas rutinarias entregando mejora de procesos, esto acentúa la importancia de unir y de interactuar con el acompañamiento de otras áreas de acuerdo a lo que se requiera para adquirir competitividad y productividad en los servicios finales.

Estas ventajas citadas se traducen en un producto con más orientación hacia el usuario y que puede promoverse velozmente a un costo reducido final.

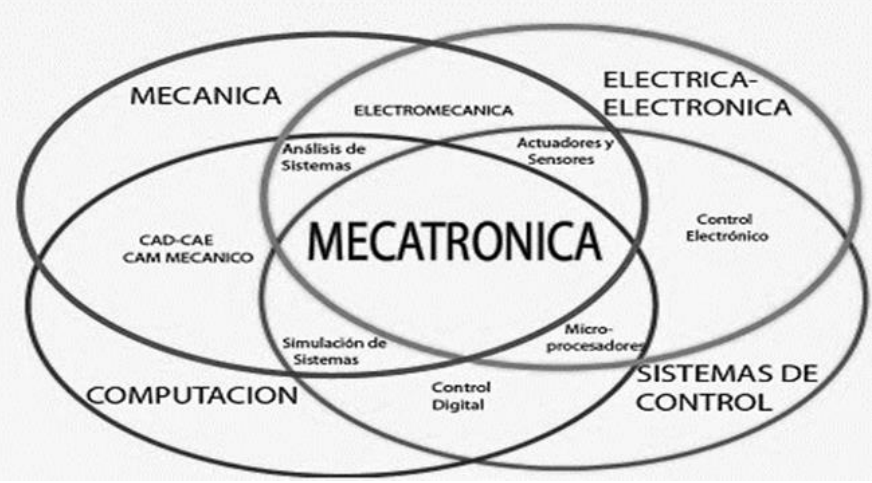

Fig. 3 Administración de Procesos Fuente (Alejandro Rodríguez. 2014) 
La Mecatrónica se la definió al principio como la integración de la mecánica y la electrónica en una máquina o producto, pero luego se consolidó como una especialidad de la ingeniería, incorporando elementos como informática, microelectrónica e inteligencia artificial. El propósito de esta "nueva ingeniería" es fabricar productos inteligentes que sean capaces de procesar información para su funcionamiento, gracias a la instalación de dispositivos y sensores electrónicos especiales.

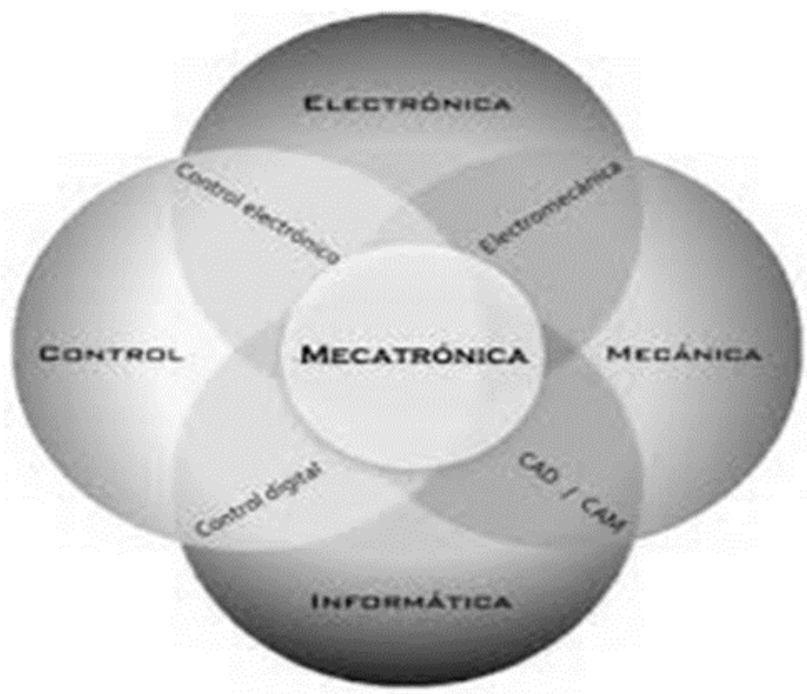

Fig. 4 Integración de elementos Fuente (Luis Sánchez. 2012)

Un sistema Mecatrónico se compone principalmente de mecanismos, actuadores, control (inteligente) y sensores. Tradicionalmente la Mecánica se ha ocupado solo de los mecanismos y los actuadores, y opcionalmente puede incorporar control. La Mecatrónica integra obligatoriamente el control en lazo cerrado y por lo tanto también a los sensores.

\section{Manufactura Integrada por Computador}

Para explicar sobre Manufactura Asistida por Computadora (CAM) es necesario referir su término Manufactura para lo cual comparto lo siguiente: Manufactura es comúnmente llamada como todo proceso de transformación de la materia, en artículos útiles para la sociedad. La manufactura tiene dos definiciones:

Sentido Amplio: Son todos los procesos que intervienen para que se dé la transformación de la materia prima a un producto terminado, desde el inicio (marketing, estudio de mercado), hasta el final (distribución de producto).

Sentido Estrecho: Es el momento en el que se le agrega valor a la materia prima, el momento en que se está transformando en producto terminado la materia prima.

Por lo tanto ya partiendo de la premisa anterior podemos comprender que la manufactura Concurrida por Ordenador frecuentemente se describe al automatismo de aplicativos de instrucciones computacionales de investigación numeraria para establecer 
ilustraciones precisas (G-code) que encaminan las máquinas de herramientas para la manufactura de partes inspeccionadas numéricamente por ordenador.

La experiencia educacional permite que la manufactura Integrada por Computador sea de gran importancia para tener el control total del proceso entero de producción, por esta razón los constructores de incomparables manufacturas ven a esto como un potencial para destacar las capacidades de CAM para producir partes de alta calidad. Un axioma más extenso de CAM consigue contener el uso de aplicativos computacionales para precisar técnicas de manufactura para el esbozo de equipos, esquema asistido por computadora (CAD) para la elaboración de modelos, sistematización, programación del reconocimiento de la máquina de medición (CMM), simulacro de máquinas de herramientas o post-procesamiento. Fig. 5 detalle de su proceso.

El procedimiento en torno a esto es ejecutado en un espacio de elaboración, control numérico directo (DNC), dirección de equipos, maquinado $\mathrm{CNC}$, o ejecución de CCM.

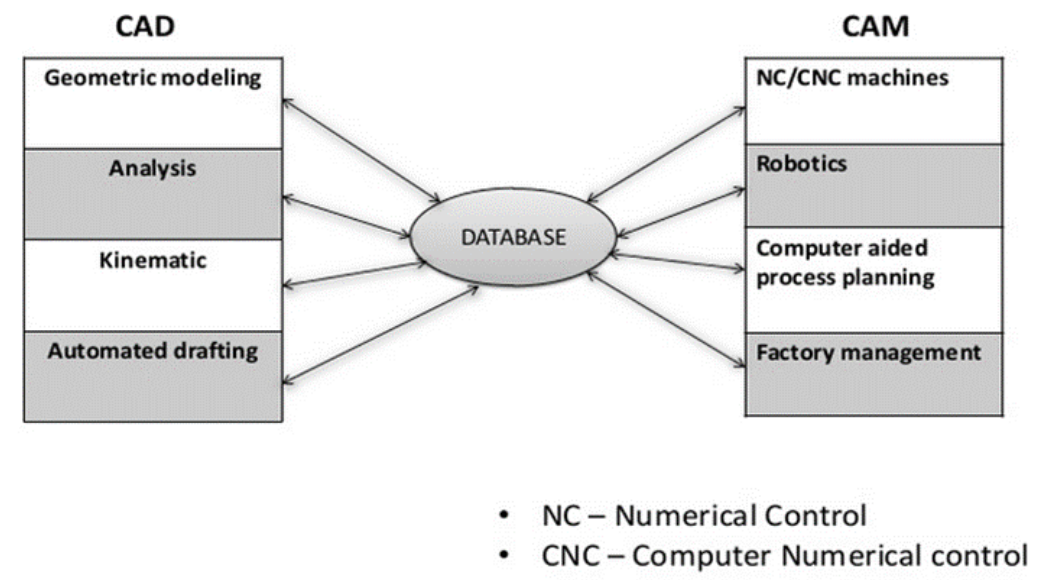

Fig. 5 Software CAD/CAM - Fuente (Krishna, 2013)

Los sistemas CAD/CAM agilizan considerablemente la operación de las máquinas CNC. Los sistemas CAD es decir Diseño Asistido por Computadora, se emplean para la fabricación de objetos y bosquejo de documentaciones, en general permite la automatización ciertas labores del ciclo del producto para hacerlas eficientes.

El CAM es decir la Manufactura Asistida por Computadora toma el objeto realizado en el CAD y crea todas las ilustraciones necesarias para que la fresadora CNC elabore y complete la pieza deseada.

\section{Estilo del Arte}

\section{Ingeniería Concurrente}

Las empresas manufactureras han sido objeto de cambios y transformaciones, resultado de la competencia por mantener y/o mejorar su posición en los mercados a nivel mundial. Las organizaciones son competitivas en la medida que mejoren la calidad de sus productos, reducen los tiempos de desarrollo de los mismos y disminuyen los costos. 
Este tipo de ingeniería genera eficiencia, cumpliendo los estándares requeridos por el cliente, tomando en cuenta costos de producción, su ciclo de vida y su calidad esté último es muy importante porque le permitirá generar la competitividad y productividad del producto final obtenido. Es considerable tomar en cuenta que las actividades mediante esta metodología se trabajan simultáneamente, pudiendo detectar errores durante el proceso de ejecución, y así mismo verificar todos los elementos del ciclo de vida del producto, esto permite tener una asertiva interacción contante, mantener una organización flexible, tomando en cuenta la perspectiva del diseño de acuerdo a las exigencias solicitadas del usuario; esto generará la disminución de tiempos de desarrollo de los productos y aumento de la flexibilidad, utilizando de manera adecuada los recursos y formando productos de alta calidad.

La metodología desarrollada se ha denominado «Desarrollo integrado del producto, un esfuerzo sistemático para mejorar la Ingeniería de Producto/Proceso a través de la conformación de equipos multidisciplinarios de trabajo (DIP/IPP)».

Está orientada a las pequeñas y medianas empresas (PYME), y las técnicas, herramientas y los enfoques de apoyo utilizados en ella pueden variar dependiendo de la empresa en la cual se va a implementar.

Se destacan como características:

-El uso de indicadores en cada etapa de la propuesta.

-Una alternativa para modelamiento dinámico del Proceso de Desarrollo del Producto.

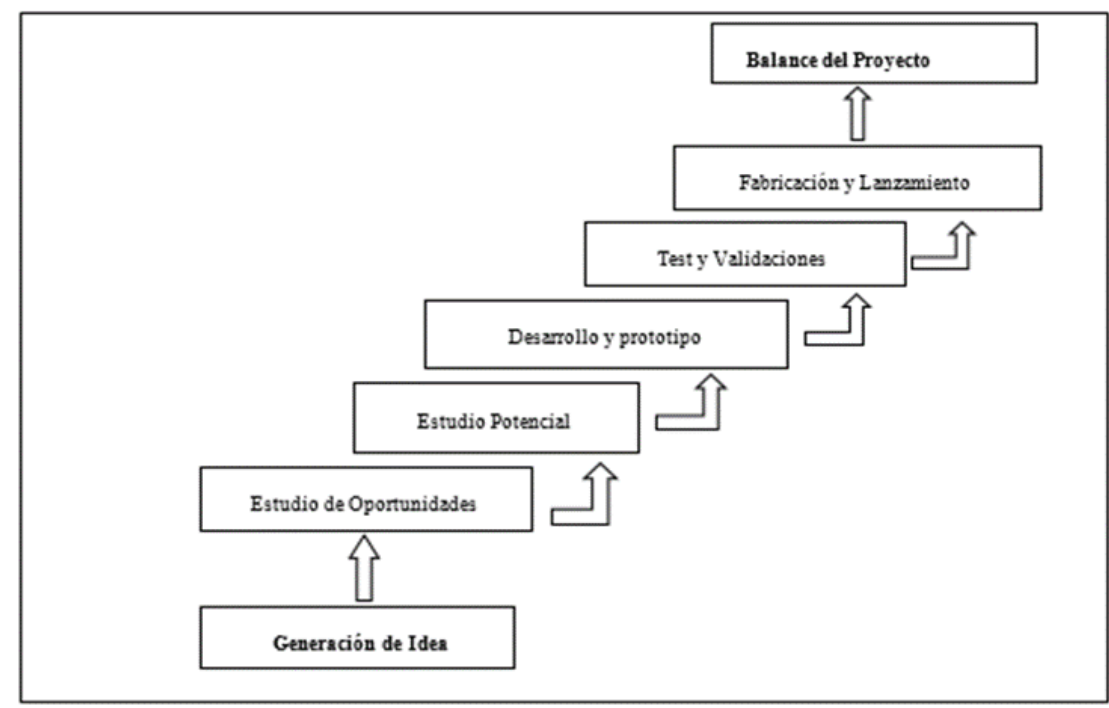

Fig.6 Descripción de Metodología

Fuente (Estela Macas. 2017)

Ejemplificando el estilo del arte de la ingeniería concurrente también puedo compartir lo siguiente: 
En contraposición al sistema tradicional, donde un diseñador entrega sus resultados a un ingeniero y éste a un matricero para que fabrique el molde o cabezal, la ingeniería concurrente centra sus esfuerzos en crear un grupo de trabajo que usa el diseño de la pieza de modo interactivo para reducir el tiempo de entrega del producto final.

En este sistema, cualquier cambio introducido por algún componente (por ejemplo, un aumento de espesor de pared por limitaciones de desempeño mecánico) es inmediatamente comunicado en forma digital al resto del grupo para que ejecute las acciones correctivas (por ejemplo, el ingeniero de moldes puede ajustar el tonelaje de cierre requerido por la máquina).

Ejemplo de prototipo conceptual:

\section{Bicicleta Back Pack.}

En las ciudades denominadas como modernas tienen problemas graves de tráfico vehicular en su centro. Para eliminar este problema se ha desarrollado todo un sistema de estacionamientos a las afueras de la ciudad que están estratégicamente ubicados (lugares sin pendientes pronunciadas) para que los trabajadores de comercios y oficinas entren a la ciudad caminando o en bicicleta.

La bicicleta convencional crearía nuevamente un problema de ordenamiento por el espacio que ocupan, por lo que se propone utilizar una bicicleta Back Pack, la cual se la puede guardar en el casillero de trabajo o en la recepción de los edificios céntricos.

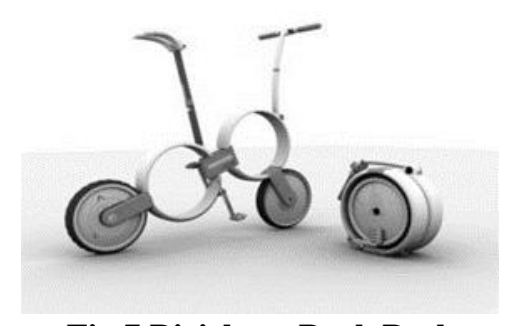

Fig.7 Bicicletas Back Pack

Fuente (Paco Condor. 2010)

Se propone un modelo que se puede transformar en un paquete y se puede guardar en un casillero de $50 \mathrm{~cm}$ x $30 \mathrm{~cm}$ x 30, las ruedas se pueden doblar por medio de una articulación y quedan concéntricas al mismo chasis de la bicicleta.

La idea, en principio, parece factible pero no hay ninguna garantía de que funcione. Se sugiere que se construya un prototipo conceptual.

Se debe tomar en cuenta que esta bicicleta tiene un concepto totalmente diferente al convencional. En su sistema de transmisión (no tiene cadena), la tracción se la realiza por fricción entre la rueda trasera y el chasis, por medio de un tren de engranes que se acopla a los pedales. Se procede a diseñar y construir el prototipo a escala natural y se consigue que el modelo funcione, el proyecto continuo y la bicicleta Back Pack completada. 


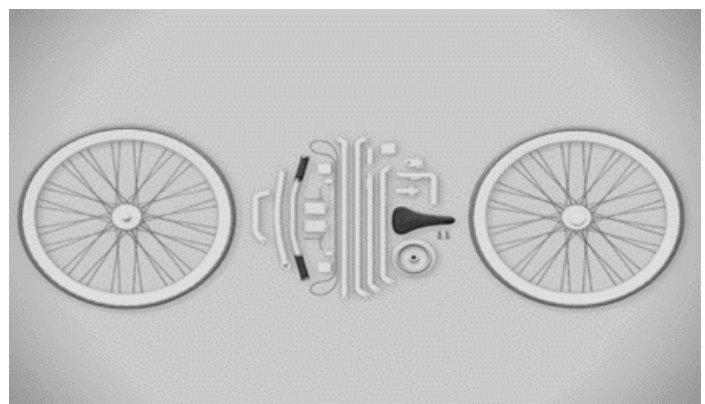

Fig.8 Partes de la Bicicleta

Fuente (ITI en Bonzi, 2017)

En este ejemplo podemos ver que no existen máquinas de referencia en el estado del arte, por lo tanto, es un prototipo conceptual. Para su construcción se ha utilizado técnicas de rapid prototyping (impresión 3D- sinterizado).

a) Estado del arte: La evolución e innovación de la bicicleta.

Desde el primer modelo visto en el año de 1970, incluso llegar a la noción actual las bicicletas sigue evolucionando, mostramos a continuación un cuadro con sus representaciones:

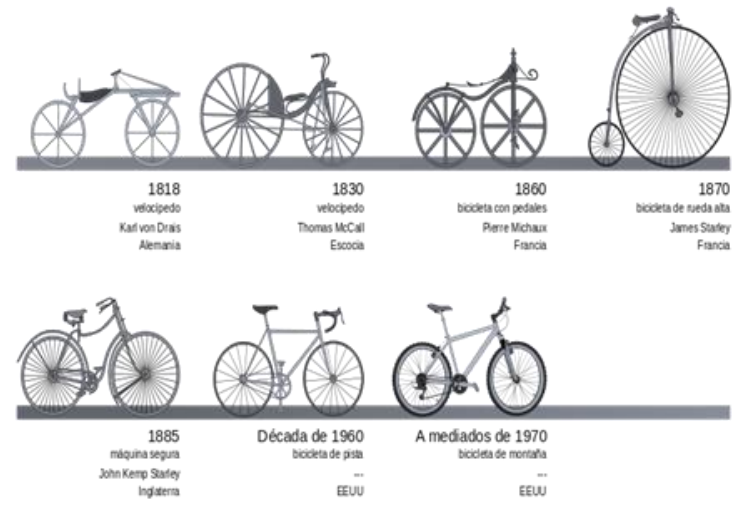

Fig.9 Evolución de Bicicletas Fuente (Richard Santana, 2016)

De acuerdo a su histórico evolucionario se puede mostrar que las figuras han ido variando en sus materiales pero muy pocos cambios en su estructura inicial, cada vez el utilizar una bicicleta nos permite mejorar cambios notables en la salud, la bicicleta se ha destinado para concienciar el apoyo al ámbito deportivo y se las ha creado de acuerdo al uso y para aportar a un inmenso grupo de condición física, entre las más destacables tenemos: bicicletas plegable, reclinada, tándem, para discapacitados.

El impacto que ha tenido en la sociedad es muy importante puesto que permite el transporte y movilidad de forma sencilla y económica, este es uno de los medios de transporte muy utilizados en el mundo entero, por su facilidad y la no contaminación al medio ambiente puesto que no utiliza ningún combustible, la tecnología está avanzando de manera significativa y su aporte al mejoramiento social es relevante, se podría pensar que en un futuro vendrán cambios 
en este medio de transporte y que se refleje una mayor calidad, resistencia, livianas y con instrumentos de navegación.

\section{Prototipo de Referencia:}

Para este ejemplo se toma como máquina de referencia la bicicleta de montaña fabricada en 1970 (se supondrá que es la más moderna hasta la fecha).

Se analiza la arquitectura de la bicicleta y se determina los principales sistemas que la componen:

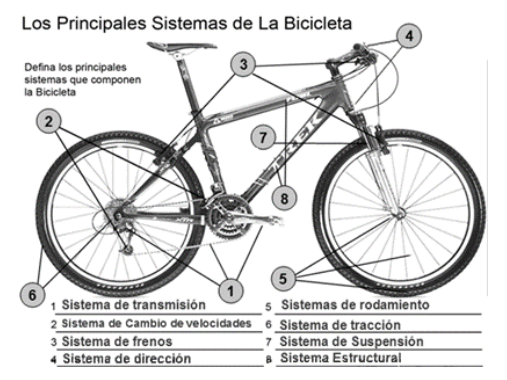

Fig.10. Descripción en el armado de la Bicicleta.

Fuente (Jaime Corzo, 2014)

De la bicicleta de montaña fabricada 1970 se determinan 8 sistemas, de los cuales se tiene que documentar la siguiente información:

- Función de cada sistema,

- $\quad$ Planos, (norma INEM),

- Material de utilizado en la fabricación de las piezas,

- Número de piezas de cada sistema,

- Peso total,

- Ajustes y tolerancias en la fabricación,

- Acabados superficiales y color,

- Lista de elementos normalizados,

- Tipos de lubricantes utilizados, etc.

Finalmente se desarrollan las especificaciones y se identifica el sistema o la pieza que puede ser mejorada o innovada. La innovación puede ser un requerimiento directo de los clientes.

\section{Mecatrónica}

La Mecatrónica es una metodología de diseño en donde se integran de manera armónica elementos mecánicos, electrónicos, programación y la robótica que es una aplicación interactiva muy particular en este ámbito. Mediante la mecatrónica podemos tener la habilidad de conjugar destrezas observando y controlando procesos; permitiéndonos interactuar aspectos interesantes como la precisión, el proceso energético en todo su sistema y el proceso mecánico de los equipos participantes. 
Un sistema mecatrónico contiene en general sensores (dispositivos que permiten medir y cuantificar las variables como la posición, la temperatura, etc), actuadores (elementos que actúan movimiento como los ventiladores, motores, etc) y los sistemas de control (sistemas que integra todos los elementos para una función adecuada)

La mecatrónica tiene varias disciplinas conformada por: la mecánica, electrónica y sistemas; por ejemplo un carro contiene estas tres áreas; en fin la mecatrónica lo que nos permite es mostrar la forma de interacción de las disciplinas con el fin de solventar y solucionar problemas y aportar a la sociedad con elementos integrados que permitan facilidad en la ejecución de tareas cotidianas, retomando el ejemplo del vehículo facilita la movilidad de un lugar a otro, minimización de tiempo y rapidez de servicio.

En el ámbito académico es importante trabajar en proyectos integradores que es la construcción de máquinas con procesos automatizados para ejecutar tareas simplificadas de acuerdo a las necesidades y tendencias actuales, estos elementos integradores otorgan procesos de mejora en su entorno y permiten la construcción de innovación tecnológica mediante los algoritmos de control, etc.

Para ejemplificar podemos poner como ejemplo un ventilador inteligente que se lo utiliza en el área médica, este dispositivo posee accesos para aire y oxigeno acoplados a una superficie en donde estos elementos se unen; adicional posee un sistema de control y una servo válvula, luego se procede a inyectar a un estado adecuado de presión y volumen. Así mismo este dispositivo cuenta con sensores que sirven como indicadores para su uso en las operaciones; el ventilador inteligente cuenta con una interfaz gráfica que sirve para establecer el comportamiento actual.

Este dispositivo de ventilador inteligente es idóneo para la toma de decisiones, puede proveer aire, muy utilizado en terapias intensivas de pacientes que tienen dificultades de espiración ya sea esto por procedencia de alguna privación fisiológico, o también utilizado en pacientes que se encuentran sosegados en quirófano porque se someterán a alguna cirugía.

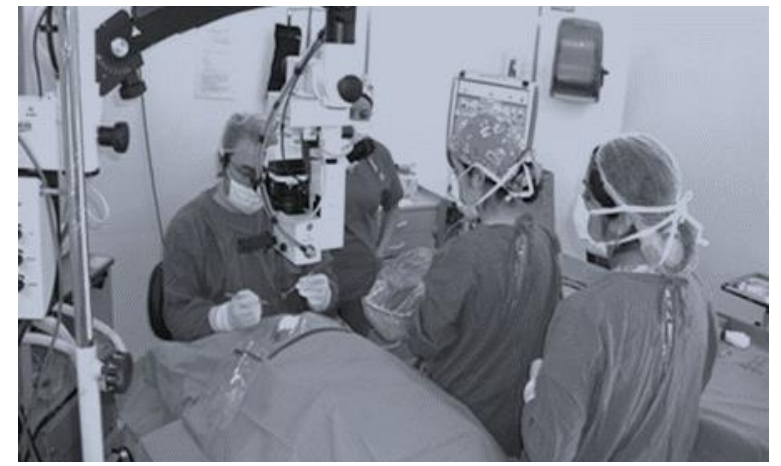

Fig.11. Utilización del Ventilador.

Fuente (Tvazteca, 2011)

Este dispositivo permite salvaguardar la vida del paciente que sufre de problemas de respiración y requieren de un ayuda mecánica para lograr una respiración adecuada, por medio de este instrumento le permite controlar el volumen, la presión, la temperatura y más aún en la 
concentración de oxígeno que debe proveer adecuadamente para que se logre una excelente ventilación.

"A diferencia de los ventiladores convencionales, que requieren que el médico o un técnico especializado seleccione el modo de ventilación más adecuado para el paciente, el ventilador inteligente selecciona automáticamente, y de acuerdo con las condiciones fisiológicas de aquél, una ventilación por presión, volumen o flujo" (Serafín Castañeda, Departamento de Ingeniería Mecatrónica).

Otro ejemplo de sistema mecatrónico es un sistema automático industrial como un PLC que de entre las ventajas existentes posee ejercer una repetición permanente, niveles de calidad óptimos, ahorro de costes, tiempos de mejoramiento en producción, seguridad confiable del personal, producción de entregas flexibles, mejora del flujo de datos existentes, y la obtención de una ventaja competitiva a pesar de todas estas singularidades aún se trabaja en las desventajas históricas entre ellas se encuentran un elevado coste de inversión, obligatoriamente ejerce una dependencia tecnológica, falta de un personal especializado y competitivo para ejecutar acciones correctas y pueda obtenerse un grado alto en su producción y competitividad.

Así mismo la coexistencia de obsolescencia tecnológica es decir tomar en cuenta que en el plan inicial se considere el desembolso de la inversión, este tipo de sistemas mecatrónicos son dispositivos pre programados utilizados para la resolución de problemas de sucesiones en la maquinaria ahorrando costos en el mantenimiento y el aumento de seguridad de los equipos o maquinaria, este tipo de dispositivo permite operar de acuerdo a su mando, detección, diálogo hombre máquina, y programación ejecutable esto sin duda alguna mejora la eficiencia y confiabilidad de los equipos.

\section{Manufactura Integrada por Computador}

Representa la automatización total de un entorno de manufactura, la versatilidad existente en esta disciplina permite cubrir las necesidades existentes, como herramienta educacional se encamina con programas orientados a la automatización, y simulación de software, aplicaciones de producción, administración de sistemas de procesos de producción, permite equilibrar disciplinas autónomas, programas interdisciplinarios enfocados en la integración de tecnologías múltiples, el proceso de manufactura integrada por computador posee varios componentes claves como un proceso sistemático de almacenamiento, es decir contar con una estación de trabajo automatizada, por ejemplo un robot colaborativo que aporte en la realización de tareas de acuerdo al proceso a ejecutar, este tipo de robot posee una estructura mecánica con la peculiaridad de poseer movimientos sincronizados cada vez repetitivos, entre otro de los ejemplos que se pueden mencionar están las maquinarias inteligentes, de control numérico estos agrupados mediante PC es una forma de perfeccionar la manufactura práctica, en esto se denota una gran coyuntura y progreso del control industrial.

La automatización es parte de la era actual, y ha proporcionado apoyo enormemente a la industria, la manufactura integrada por computadora logra automatizar y optimizar por medio de PC los procesos existentes; y propone la utilización de análisis, cálculo, desarrollo de software, 
para contribuir al mercado competitivo. Las aplicaciones construidas deben ser funcionales, óptimas, con criterio de apoyo a la toma de decisiones del proceso en bien de la organización.

\section{Relaciones y Diferencias}

\section{Relación entre Ingeniería Concurrente y Manufactura Integrada por Computador}

La manufactura asistida por computador y la ingeniería concurrente son actividades dirigidas a mejorar el diseño y las especificaciones de los productos a lo largo de las fases de investigación, desarrollo, diseño y producción.

Estas actividades traen muchos beneficios. Entre ellos, pueden mencionarse los siguientes:

$\checkmark$ Reducción de costos.

$\checkmark$ Reducción de la complejidad de los productos.

$\checkmark$ Mejora de los aspectos funcionales del producto.

$\checkmark$ Mejora del diseño del trabajo.

$\checkmark$ Mejora del mantenimiento del producto.

Existen estudios que indican que un peso gastado en estas actividades puede traer ahorros desde 10 hasta 25 veces económicamente hablando en procesos posteriores, por lo que se considera sumamente importante dedicar tiempo y recursos suficientes a estas actividades.

El diseñador puede, sobre su puesto de trabajo, crear la pieza a diseñar con su modelizado de sólidos. A su vez, basándose en herramientas de diseño asistido por computador, puede generar los planos detallados en dos dimensiones de la pieza, planos que pueden ser analizados y corregidos por un tercero, que puede ser el cliente, el responsable de fabricación o el responsable de montaje, o mejor todos a la vez.

Asimismo, en base a herramientas informáticas, el diseñador puede, sobre su propio puesto de trabajo, simular el proceso de fabricación con herramientas de control numérico, y el montaje, por ejemplo robotizado, de la pieza.

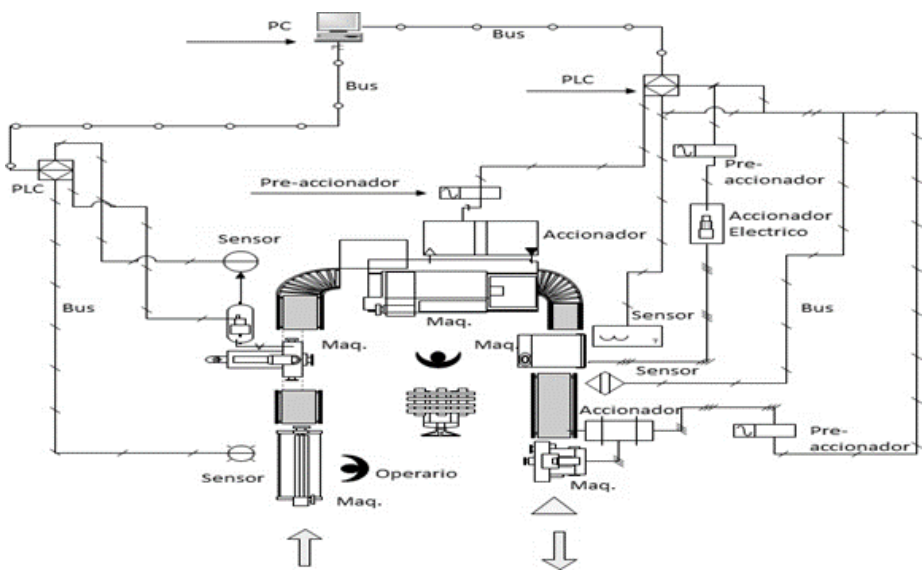

Fig. 12 Utilización de Sistema Computarizado

Fuente (Alexander Ramos, 2012) 
Por ejemplo en el campo del montaje todavía se debe localizar otro factor importante en la idea de la concurrencia en la transmisión de información de diseño.

Si en la fase de montaje, simulado por supuesto, se detecta un problema que afecta a más de una pieza, por ejemplo un ajuste, la modificación introducida, que afecta a varias piezas, debe ser capaz de ser procesada en todas de una manera automática, sin obligar al diseñador a recordar y localizar cuáles son las piezas a las que esta modificación pueda afectar.

Se gestionan las modificaciones de manera automática, sin intervención exterior, único elemento que garantiza que el conjunto guarda su integridad y su coherencia intrínseca. Se hace necesario, por tanto, la utilización de los ordenadores y de los sistemas informáticos como herramientas habituales de diseño.

\section{Relación entre Ingeniería Concurrente y Mecatrónica}

Utilizan una serie de principios, los cuales son empleados en un enfoque sistematizado y están relacionados con la introducción de cambios culturales, organizacionales, y tecnológicos en las compañías, a través de una serie de una serie de metodologías, técnicas y tecnologías de información.

Los objetivos globales que se persiguen con la implementación son:

$\checkmark$ Acortar los tiempos de desarrollo de los productos.

$\checkmark$ Elevar la productividad.

$\checkmark$ Aumentar la flexibilidad.

$\checkmark$ Mejor utilización de los recursos.

$\checkmark$ Productos de alta calidad.

$\checkmark$ Reducción en los costos de desarrollo de los productos.

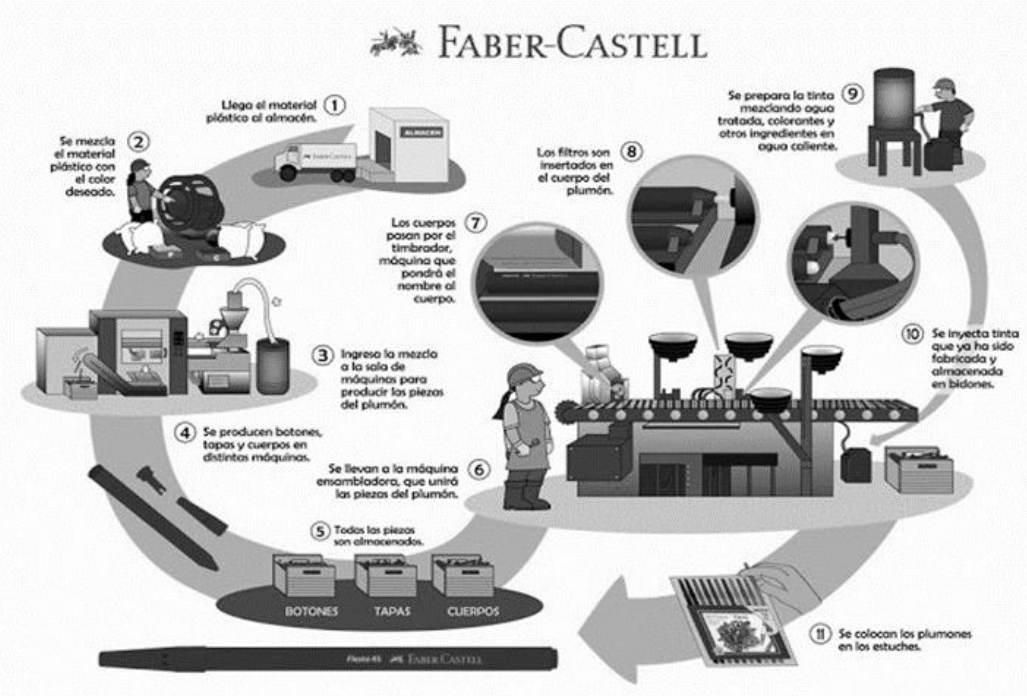

Fig. 13 Relación aplicada en la Ingeniería Industrial Fuente (Diego Aranda, 2011) 


\section{Relación entre Manufactura Integrada por Computador CAM y Mecatrónica}

La relación radica en que el proceso digital permite implementar estrategias de control y supervisión de variada naturaleza a partir de una misma configuración. Permite realizar control de posición y velocidad de una manera muy precisa y eficiente. Los robots y las máquinas son básicamente mecanismos que no pueden funcionar si no es por el computador que hace el control de posicionamiento local y la coordinación de los mismos.

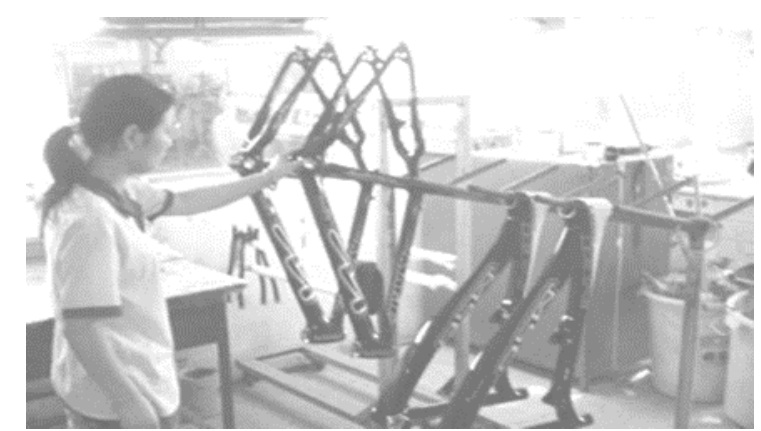

Fig.14 Relación de CAM y Mecatrónica Fuente (Elizabeth Hernández, 2017)

\section{Conclusiones}

Para que un país pueda producir en forma competitiva productos manufacturados exportables, es necesario generar una fuerte capacidad de Diseño y Desarrollo de Productos, que abarque desde la concepción hasta la industrialización. De este modo las empresas pueden realmente introducir, en el debido momento, nuevos productos en mercados globalizados muy dinámicos.

Para esto se debe articular un proceso o metodología adecuada a la realidad nacional, que sea replicable a un amplio grupo de productos, y que sea económico y rápido. Dicho proceso debe contemplar una serie de actividades, cada una de las cuales debe realizarse de una manera óptima empleando las herramientas tecnológicas más avanzadas disponibles, con esto lo que se logrará es innovación y un país con innovación es un país que se apunta directamente a crecer su status en toda índole, ya que optimiza y consigue productos con procesos tecnológicos viables.

Así mismo en la parte industrial con más precisión la innovación se ha propiciado principalmente por el "empuje tecnológico" más que por la demanda de los mercados globales crecientes de los procesos industriales.

Para poder cumplir con esta dinámica los fabricantes han tenido que recurrir a instalar y trabajar bajo combinaciones de los modelos de innovación más actuales, tales como la mecatrónica, manufacturación y los modelos de producción por ingeniería concurrente

\section{Criterio Personal}

Una vez analizados las temáticas como Mecatrónica, Manufactura integrada por computador e Ingeniería Concurrente vemos de manera significativa como coadyuvan al mejoramiento de procesos en el entorno industrial, médico, académico y otros entornos afines 
para contribuir al mejoramiento del desarrollo local, nacional e internacional por cuanto son áreas de progreso para el engrandecimiento fructífero de la sociedad.

Estos tópicos nos permitirán de manera general establecer, plantear, edificar, elaborar, conservar, administrar eficientemente los procesos desde una perspectiva automatizada y a raíz de estas premisas investigar futuras innovaciones de emprendimiento para la calidad de vida exitosa en el ámbito industrial sustentable y contribuir positivamente a la sociedad en el entorno que se requiera, estas temáticas afirman notablemente la superación eficaz en la gestión asertiva de las organizaciones.

\section{Bibliografía}

Aranda, D. (2011). Ejemplo De Un Proceso De Manufactura. Industrial En Tu Secundaria Recuperado De: Http://Www.Portaldeingenieria.Com/Home/

Carvajal, J. (2008). Automatización Y Robótica Y Su Impacto En La Productividad Empresarial. Universidad De La Salle, Bogotá. Recuperado De: Http://Lattes.Cnpq.Br/9921036582975891

Carvallo. E (2014). La Gestión Por Procesos. Universidad Peruana De Ciencias Aplicadas. Departamento Administrativo. Almacenes.

Cóndor, P (2010). Bicicletas Plegables Súper Modernas. Cinco Bicicletas Plegables Super Modernas. La Bicicleta No Contamina... Úselo Recuperado De: Http://Andoenbicicletaynocontamino.Blogspot.Com/2010/12/Bicicletas-Plegables-SuperModernas.Html

Córdova. E (2010). Automatización Y Reponteaciación Tecnológica Del Proceso Industrial Del Control De Calidad De Engranajes. Universidad Nacional De Colombia Isbn: 9789587619300 .

Corzo, J. (2014) Partes De Una Bicicleta Recuperado De: Https://Ugc.Kn3.Net

Domínguez, C. A. (2012) Cosas De Mecatrónica Entrevista Pionero De La Robótica. Recuperado De: Https://Mecatronica.Wordpress.Com/Page/3/

Domínguez, L. (2014). Sistemas De Gestión Integrada Para Las Empresas: Erp Servicios De Publicaciones.

Flexsim (2014). Software Flexsim 3d Simulation. User Manual Version Flexsim 7.1.2 S. L Recuperado De: Https://Www.Flexsim.Com

Gramegna, N.; Della, E.; Poles S (2011). Manufacturing Process Simulation for Product Design Chain Optimization. Padova.

Grupo Lexnova (2011). La Gestión Del Proceso De Negocio. Recuperado De: Http://Tecnojur.Blogs.Lexnova.Es/Files/2011/03/Ciclo.Gif

Hernández, E (2017). Proyectos Tecnológicos. Emprendimientos Técnicos. Unidad Educativa Pío Jaramillo Alvarado. 
Jacobson, I. (2013). La Esencia De La Ingeniería De Software: El Núcleo De Semat. Latinoamericana De Ingeniería De Sotware, 1.

Jaramillo, M. \& Gutiérrez, R. (2000). Implantación De La Filosofía De Ingeniería Concurrente Para El Desarrollo De Nuevos Productos En Una Industria De Productos Plásticos. Universidad Eafit Departamento De Ingeniería De Producción. Medellín (Colombia)

Krishna, K (2013). Mecanizado Cad/Cam/Sim. Cultura Académica Recuperado De: Https:/Www.Slideshare.Net/Krishnakanth12/Cad-And-Cam

Ktistakis. T. Sales M. A. (2004). Solución Por Función De Negocios. Recuperado De: Http://Www.Plm.Automation.Siemens.Com/

Notimex (2011) Desarrollan En La Unam Ventilador Médico. Tv Azteca Http://Www.Aztecanoticias.Com.Mx/Notas/Tecnologia/59903/Desarrollan-En-La-UnamVentilador-Para-Uso-Medico

Paucar, J (2015) Proyecto De Inversión. Estudio Técnico Ingeniería Del Proyecto. Universidad César Vallejo.

Pawleski, P. (2014). Management in Manufacturing. Proceedings of the 47th Cirp Conference on Manufacturing Systems, 2014

Ramos, A. (2012). Diagrama Estructura Sistema Automatizado. Recuperado De: Http://Www.Portaldeingenieria.Com/Home/

Rodríguez, A (2017). Reporte De Proyectos Con Automatización Industrial. Embotelladora Automatizada. Expo Mecatrónica. Universidad Politécnica De Querétaro. Recuperado De: Http://Expomecatronica.Blogspot.Com/

Rojas, L. M. D., \& Ruiz, R. C. (2013). Introducción A La Ingeniería. Bogotá, Co: Ediciones De La U.

Rojas, L.M.D (2015). Evaluación De Proyectos Para Ingenieros (2da. Ed). Bogotá, Co: Ecoe Ediciones.

Ruiz, N.; Giret, A.;Botti, V (2014) An Intelligent Simulation Environment For Manufacturing Systems. Computer \& Industrial Engineering, 2014, Vol. 76.

Sánchez, L. (2012). Generalidades De La Ingeniería Mecatrónica. Introducción A La Mecatrónica Recuperado De: Http://1510646.Blogspot.Com/

Santana, R. (2016) Descubre La Evolución De Las Bicicletas Desde Su Inicio Hasta Hoy. Recuperado De: Http://Pedaleandoporcanarias.Com/Wp-Content/Uploads/2016/04/2000pxBicycle_Evolution-Fr.Png 